\title{
New Orally Bioavailable 2-aminobenzamide-type Histone Deacetyase Inhibitor Promotes Neurite Outgrowth via Histone H3 Modification in PC12 cells: a Possible Therapeutic Candidate for Neuronal Diseases
}

\author{
Hiroki Maruoka',2, Ryo Hosokawa', Yoshiyuki Hirata', Hiromichi Kawa', Kouta Okamoto', Taiki Waki', Shinichi Uesato', Toshihiko Ikeuchi ${ }^{1}$ \\ and Koji Shimoke ${ }^{1 *}$
}

${ }^{1}$ Department of Life Science and Biotechnology, Faculty of Chemistry, Materials, and Bioengineering and Strategic Research Base, Kansai University, 3-3-35, Yamatecho, Suita, Osaka 564-8680, Japan

${ }^{2}$ Technology Research Laboratory, KURABO, Neyagawa, Osaka 572-0823, Japan

\begin{abstract}
Histone deacetylase (HDAC) inhibitors are known to function as anticancer agents, but they can also induce neuronal differentiation and neuroprotection in the central nervous system (CNS) and have been investigated as therapies for traumatic brain injury (TBI). A new, orally bioavailable 2-aminobenzamide-type HDAC inhibitor, $\mathrm{N}$-(2aminophenyl)-4-((2-hydroxyethyl)(4-(thiophen-2-yl)benzyl)amino)methyl)benzamide (K-350), was recently shown to inhibit the growth of HCT116 colon cancer cell xenografts in nude mice. However, the effect of K-350 on the central and the peripheral nervous system is largely unknown.

This study analyzed the effects of K-350 on a model neuron, PC12 cells. We report that Lys 14 of histone H3 was acetylated gradually in PC12 cells after exposure to K-350, with peak acetylation $2 \mathrm{~h}$ after treatment. RT-PCR experiments demonstrated that the nur77 immediate early gene expression was up-regulated $2 \mathrm{~h}$ after treatment. These results suggest that the expression of nur77, which is essential for neuronal differentiation induced by K-350, is up-regulated via the acetylation of histone $\mathrm{H} 3$ in PC12 cells.
\end{abstract}

Keywords: Differentiation; HDAC; Local chromatin structure; Immediate early gene; Nur77; PC12 cell

\section{Introduction}

Histone deacetylase (HDAC) inhibitors induce changes in gene expression and differentiation, growth arrest, and apoptosis in cancer cells, and are used clinically as a less toxic antitumor drug. For example, MS-275 is being clinically evaluated for the treatment of metastatic melanoma [1], while suberoylanilide hydroxamic acid (SAHA) is being used to treat T-cell lymphoma [2].

HDAC inhibitors have also emerged as useful therapeutic tools in other areas of clinical therapy. In neurological disorders, valproic acid (VPA) is a drug that is widely used to treat epilepsy [3]. There are also a number of reports on HDAC inhibitors as novel therapeutics in models of ischemic stroke [4-6] and Huntington's diseases [7,8]. More recently, MS-275 was shown to be a potent and selective HDAC inhibitor that crosses the blood-brain barrier [9]. MS-275 is being evaluated as a potential therapeutic drug in the brain.

There are currently few reports on the effects of HDAC inhibitors for the treatment of traumatic brain injury (TBI). Protection of neurons from apoptosis and promotion of neurite outgrowth are critical factors in the treatment of TBI [10-13]. Since HDAC inhibitors are known to function in both neuroprotection and neuronal differentiation, they have emerged as attractive potential new therapeutics for TBI.

Several HDAC inhibitors have been shown to protect against glutamate-related excitotoxicity $[14,15]$ in cultured neurons; however, data on the participation of HDAC inhibitors in neurite outgrowth is currently lacking. In one report, Inokoshi et al. [16] demonstrated that the HDAC inhibitor trichostatin A (TSA) induced neurite extension in cultured Neuro 2a cells. Also, TSA or sodium phenyl butyrate (PB) induced a significant increase in total average neurite length respectively in rat cerebella granule neurons [17]. However, details of the mechanism by which HDAC inhibitors induce neurite outgrowth remain unknown.

Kiyokawa et al. [18] recently synthesized a new 2-aminobenzamidetype histone deacetylase (HDAC) inhibitor named K-350. K-350 features both a sulfur-containing bicyclic arylmethyl moiety-a surface recognition domain, introduced to increase in cellular uptake, and a substituted tert-amino group, which influences physicochemical properties such as aqueous solubility. The investigators reported that $\mathrm{K}-350$ suppressed the growth of tumor xenografts in a manner similar to the effects observed for MS-275, a 2-aminobenzamide-type HDAC inhibitor, in a human colorectal carcinoma HCT116 xenograft model. In addition, K-350 and MS-275 decreased xenograft tumor weight to a similar degree. Further mechanistic in vitro studies revealed that both K-350 and MS-275 caused hyperacetylation of histone $\mathrm{H} 3$ and inhibited HCT 116 cancer cell growth via similar mechanisms. These data suggest that the HDAC inhibitor K-350 could become a new anticancer drug. Since mice dosed with K-350 do not show any action abnormalities in comparison with MS-275-dosed mice, it has been suggested that K-350 may prove useful as a novel neurologic therapeutic drug. However, the

${ }^{*}$ Corresponding author: Koji Shimoke, Department of Life Science and Biotechnology, Faculty of Chemistry, Materials, and Bioengineering and Strategic Research Base, Kansai University, 3-3-35, Yamate-cho, Suita, Osaka 564-8680, Japan, Tel: 81-6-6368-0853; Fax: 81-6-6330-3770; E-mail: shimoke@kansai-u.ac.jp

Received November 11, 2011; Accepted December 26, 2011; Published December 30, 2011

Citation: Maruoka H, Hosokawa R, Hirata Y, Okamoto K, Kawa H, et al. (2012) New Orally Bioavailable 2-aminobenzamide-type Histone Deacetyase Inhibitor Promotes Neurite Outgrowth via histone H3 Modification in PC12 cells: a Possible Therapeutic Candidate for Neuronal Diseases. J Bioengineer \& Biomedical Sci S5:001. doi:10.4172/2155-9538.S5-001

Copyright: @ 2012 Maruoka H, et al. This is an open-access article distributed under the terms of the Creative Commons Attribution License, which permits unrestricted use, distribution, and reproduction in any medium, provided the original author and source are credited. 
Citation: Maruoka H, Hosokawa R, Hirata Y, Kawa H, Okamoto K, et al. (2012) New Orally Bioavailable 2-aminobenzamide-type Histone Deacetyase Inhibitor Promotes Neurite Outgrowth via histone H3 Modification in PC12 cells: a Possible Therapeutic Candidate for Neuronal Diseases. J Bioengineer \& Biomedical Sci S5:001. doi:10.4172/2155-9538.S5-001

effects of K-350 in the central and peripheral nervous system and its potential as a useful therapeutic drug for the treatment of neuronal injury remain largely unknown. In this study, we report that K-350 has the ability to induce neurite outgrowth in a model neuron, PC12 cells. HDAC inhibitor-induced neurite outgrowth has been the subject of recent investigations. For example, Hao et al. [19] reported that VPA promotes neurite outgrowth, and this outgrowth is regulated by the ERK cell signaling pathway in rat cortical neurons. However, the detailed molecular mechanism driving the early phase of HDAC inhibitor-induced neurite outgrowth has yet to be clarified.

We previously conducted focused research on the early phase of dibutyryl-cAMP (dbcAMP)-induced neurite outgrowth and reported that dbcAMP-induced neurite outgrowth is regulated by immediate early gene, nur77 expression in PC12 cells [20]. Results indicated that acetylation of histone $\mathrm{H} 3$ gradually increased during the early period of differentiation, $0-1 \mathrm{~h}$ after dbcAMP treatment. Treatment also induced the expression of nur77 within $1 \mathrm{~h}$ in PC12 cells.

Regarding treatment with HDAC inhibitors, TSA is known to induce histone $\mathrm{H} 3$ hyperacetylation in rat cerebella granule neurons as early as $4 \mathrm{~h}$ after treatment [17]. However, it is still unclear whether nur77 expression is required for HDAC inhibitor-induced neurite outgrowth in PC12 cells.

Using PC12 cells as a model system, the present study first evaluated whether K-350 could induce neurite outgrowth, and then investigated the mechanism by which nur77 gene expression is regulated during neurite outgrowth induced by K-350. Results indicated that nur77 is upregulated during neurite outgrowth induced by K-350 in PC12 cells, suggesting that the nur77 gene product is important for neuronal differentiation which may be a useful target for treatment of TBI.

\section{Materials and Methods}

\section{Compounds}

$\mathrm{N}$-(2-aminophenyl)-4-((2-hydroxyethyl)(4-(thiophen-2-yl)benzyl)amino)methyl)benzamide (K-350) and MS-275 were synthesized by Prof. Uesato's group.

\section{Cell culture}

PC12 cells were maintained in Dulbecco's Modified Eagle's Medium (DMEM) supplemented with 5\% (v/v) fetal bovine serum (FBS), $5 \%(\mathrm{v} / \mathrm{v})$ heat-inactivated horse serum, and $0.1 \%(\mathrm{v} / \mathrm{v})$ penicillinstreptomycin solution (Gibcco BRL).

\section{Measurement of cell viability}

Viable cells were quantified using an MTT assay. Briefly, cells were plated in a 96-well plate at $1 \times 10^{4}$ cells $/ \mathrm{cm}^{2}$ and cultured in DMEM supplemented with $5 \%(\mathrm{v} / \mathrm{v})$ fetal bovine serum (FBS), $5 \%(\mathrm{v} / \mathrm{v})$ heat-inactivated horse serum, and $0.1 \%(\mathrm{v} / \mathrm{v})$ penicillin-streptomycin solution. Then, the cells were treated with $0,0.06,0.18,0.56,1.67$ and $5 \mu \mathrm{M}$ of either K-350 or MS-275 in serum free medium supplemented with $0.1 \%(\mathrm{v} / \mathrm{v})$ penicillin-streptomycin solution for 24 or $48 \mathrm{~h}$. After treatment, the medium was changed again to SF-DMEM containing 5\% (v/v) MTT solution and cells were incubated for $2 \mathrm{~h}$. Cell fluorescence intensity was detected using a spectrophotometer at $750 \mathrm{~nm}$. Cell viability was defined as [(test sample count) - (blank count)/(untreated control count) - (blank count)] x 100.

\section{Measurement of neurite outgrowth}

PC12 cells were plated in 24 -well plates at $0.2 \times 10^{4}$ cells $/ \mathrm{cm}^{2}$ and cultured in DMEM supplemented with 5\% (v/v) fetal bovine serum (FBS), 5\% (v/v) heat-inactivated horse serum, and $0.1 \%(\mathrm{v} / \mathrm{v})$ penicillin-streptomycin solution. Adherent cells were treated with 0 , $0.06,0.18,0.56,1.67$ and $5 \mu \mathrm{M} \mathrm{K}-350$ in DMEM supplemented with $1 \% \mathrm{FBS}$ and $0.1 \%(\mathrm{v} / \mathrm{v})$ penicillin-streptomycin solution for $24 \mathrm{~h}$. After the treatment, the cells were fixed with $4 \%$ paraformaldehyde for 30 min. For analysis of neurite outgrowth, cells (200 cells/well) were randomly photographed using a fluorescence microscope (Biozero BZ9100, KEYENCE, Osaka, Japan). Neurite lengths were measured using the BZ-H1C software (KEYENCE). Differences between groups were examined for statistical significance using the Kolmogorov-Smirnov test. A P value less than 0.05 denoted the presence of a statistically significant difference.

\section{Immunoblot analysis}

PC12 cells were seeded onto 6-cm diameter dishes and the medium was changed to serum-free DMEM. After incubation for $16 \mathrm{~h}$, cells were either left untreated or were treated with $5 \mu \mathrm{M}$ of $\mathrm{K}-350$ for 0,1 , 2 or 4 h. Cells were then lysed in a lysis buffer containing $50 \mathrm{mM}$ Tris$\mathrm{HCl}$ (pH7.8), $150 \mathrm{mM} \mathrm{NaCl}, 1 \%$ sodium dodecylsulfate (SDS), $1 \mathrm{mM}$ ethylenediaminetetraacetic acid (EDTA), $1 \mathrm{mM}$ phenylmethylsulfonyl fluoride (PMSF), $2 \mu \mathrm{g} / \mathrm{ml}$ leupeptin, $2 \mu \mathrm{g} / \mathrm{ml}$ of aprotinin, $1 \mathrm{mM}$ $\mathrm{Na}_{3} \mathrm{VO}_{4}$, and $10 \mathrm{mM}$ sodium butyrate [21]. Cell lysates $(30 \mu \mathrm{g}$ of protein per lane) were loaded on a sodium dodecylsulfate-polyacrylamide (SDS-PAGE) gel. Following separation by electrophoresis in Laemmli buffer, proteins were transferred onto a PVDF membrane using a semidry blotter (Atto, Japan). Membranes were blocked in 5\% skimmedmilk (Nakarai, Japan), incubated with either a monoclonal anti-histone $\mathrm{H} 3$ antibody (Upstate Biotechnology) or an anti-acetylated histone H3 (Lys14) antibody (Upstate Biotechnology), and after washing were incubated with the appropriate horseradish peroxidase-conjugated second antibody (Santa Cruz). Membrane-bound antibodies were detected using enhanced chemiluminescence (SuperSignal West Femto Maximum Sensitivity Substrate; Pierce, USA) and visualized with a light-capture system (Atto, Japan).

\section{RNA preparation and RT-PCR}

PC12 cells were seeded onto $6 \mathrm{~cm}$ diameter dishes and the medium was changed to serum-free DMEM. After incubation for $16 \mathrm{~h}$, the cells were either left untreated or were treated with $5 \mu \mathrm{M} \mathrm{K}-350$ for $0,1,2$ or $4 \mathrm{~h}$. Total RNA was collected using Isogen (Nippongene) followed by extraction with phenol-chloroform. cDNA was synthesized from the purified total RNA using the ReverTra Ace ${ }^{\circledR}$ qPCR RT Kit (TOYOBO), PCR primers for nur77 (sense: CCGGTGACGTGCAGCAATTTTATGAC antisense: GGCTAGAATGTTGTCTATCCAGTCACC) and beta-actin (sense: TGTGATGGACTCCGGTGACGG antisense: ACAGCTTCTCTTTGATGTCACGC) were synthesized as previously described [23]. cDNAs were amplified using TaKaRa Taq polymerase for 30 cycles.

cDNA levels were expressed semiquantitatively based on the total area of white pixels. Pixel intensity was determined using CS Analyzer 1.0 software (Atto, Japan).

\section{Results}

Initial experiments evaluated K-350 toxicity in PC12 cells. For this experiment, PC12 cells were plated and treated with $0,0.06,0.18,0.56$, 1.67 or $5 \mu \mathrm{M}$ of K-350 for 24 or $48 \mathrm{~h}$ (Figure 1). Results confirmed that $24 \mathrm{~h}$ exposure at up to $5 \mu \mathrm{M}$ of K-350 did not induce cell death in nearly all PC12 cells. However, treatment with $5 \mu \mathrm{M}$ of K-350 for $48 \mathrm{~h}$ did induce PC12 cell death (Figure 1). The potential toxicity of MS-275, 
Citation: Maruoka H, Hosokawa R, Hirata Y, Kawa H, Okamoto K, et al. (2012) New Orally Bioavailable 2-aminobenzamide-type Histone Deacetyase Inhibitor Promotes Neurite Outgrowth via histone H3 Modification in PC12 cells: a Possible Therapeutic Candidate for Neuronal Diseases. J Bioengineer \& Biomedical Sci S5:001. doi:10.4172/2155-9538.S5-001

Page 3 of 5

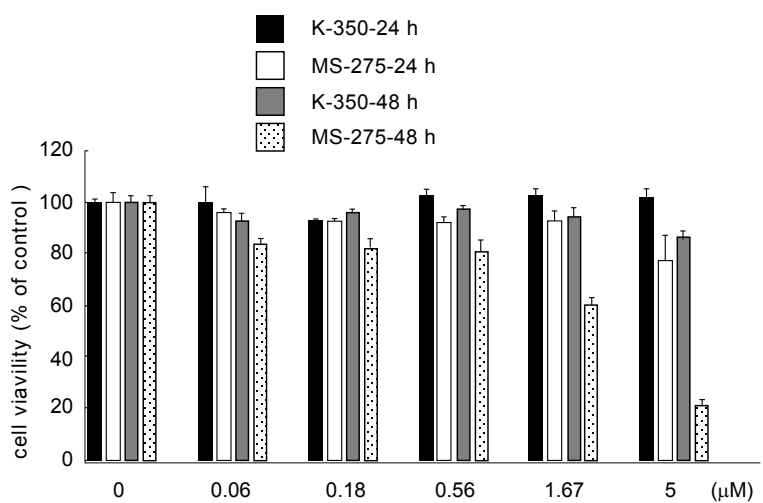

Figure 1: Measurement of cell viability in PC12 cells treated with either K-350 or MS-275.

PC12 cells were untreated (con) or were treated with $0,0.06,0.18,0.56,1.67$ or $5 \mu \mathrm{M}$ of either K-350 or MS-275 for 24 (hours) h (black bars and gray bars, respectively) or $48 \mathrm{~h}$ (white bars and dot bars, respectively). Viable cells were quantified using an MTT assay.

A
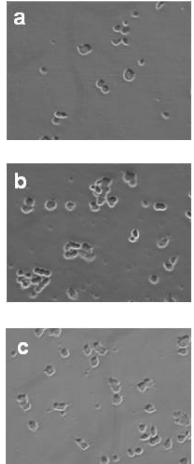

B

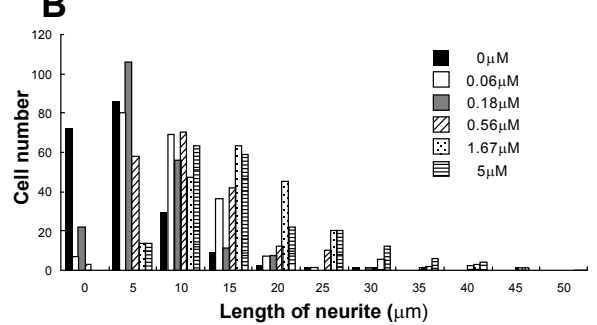

Figure 2: K-350-induced PC12 cell neurite outgrowth

(A) Photomicrographs of PC12 cells treated with K-350. PC12 cells were cultured for $24 \mathrm{~h}$ with (a) 0, (d) 0.06, (b) 0.18, (e) 0.56, (c) 1.67 and (f) $5 \mu \mathrm{M}$ K-350. Scale bars: $50 \mu \mathrm{m}$.

(B) Histogram of neurite lengths for PC12 cells treated for $24 \mathrm{~h}$ with $0,0.06$, $0.18,0.56,1.67$ and $5 \mu \mathrm{M} \mathrm{K}-350$ (black, white, gray, stripe, dot and horizontal stripe). Neurite length was measured using the BZ-H1C software.

a similar 2-aminobenzamide-type HDAC inhibitor, was also assessed in PC12 cells. PC12 cells were plated and treated with MS-275 at the same concentrations as K-350 for 24 or $48 \mathrm{~h}$ (Figure 1). The results also confirmed that treatment with $5 \mu \mathrm{M}$ of MS-275 caused minimal cell death and nearly all PC12 cells remained viable after $24 \mathrm{~h}$ in culture. However, treatment with MS-275 at concentrations greater than 1.67 $\mu \mathrm{M}$ resulted in PC12 cell death after $48 \mathrm{~h}$ in culture. These results suggest that both K-350 and MS-275 at $5 \mu \mathrm{M}$ are not toxic to PC12 cells after 24 $\mathrm{h}$ in culture. We next examined whether K-350 at $5 \mu \mathrm{M}$ was sufficient to induce neurite outgrowth in PC12 cells. Measurement of the length of neurites produced $24 \mathrm{~h}$ after treatment with $5 \mu \mathrm{M} \mathrm{K}-350$ confirmed that K-350 promoted neurite outgrowth and K-350-induced neurite outgrowth was dose-dependent in PC12 cells (Figure 2). At $5 \mu \mathrm{M}$, $\mathrm{K}-350$ induced remarkable neurite outgrowth which was statistically significant when compared with untreated cells. These results suggest that K-350 induces neurite outgrowth in PC12 cells.

Additional analyses evaluated whether treatment of PC12 cells with $5 \mu \mathrm{M}$ of $\mathrm{K}-350$ was sufficient to induce histone $\mathrm{H} 3$ acetylation. Immunoblots probed for acetylation of Lys 14 of histone $\mathrm{H} 3$ confirmed that $\mathrm{K}-350$ at $5 \mu \mathrm{M}$ can induce acetylation of Lys 14 of histone $\mathrm{H} 3$ after $2 \mathrm{~h}$ of exposure (Figure 3 ). This result also suggests that in PC12 cells, acetylation of Lys14 of histone $\mathrm{H} 3$ is regulated by $\mathrm{K}-350$ during the early period of neurite outgrowth, $0-4 \mathrm{~h}$ after the treatment.

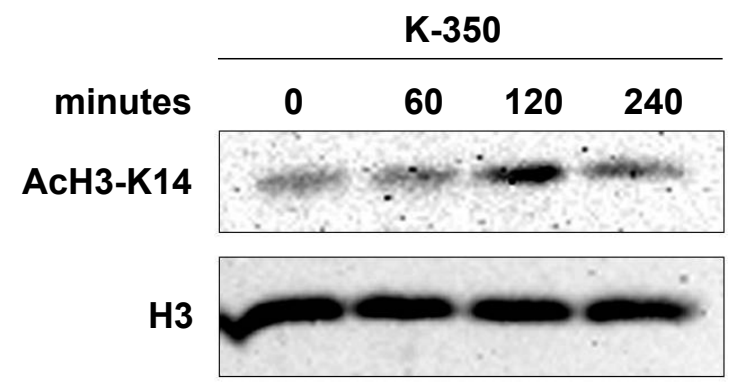

Figure 3: Effect of K-350 on the acetylation of Lys 14 on histone $\mathrm{H} 3$ in PC12 cells.

Western blot analysis of K-350-induced acetylation of Lys 14 on histone $\mathrm{H} 3$ in PC12 cells. Cells were cultured in serum-free DMEM were either untreated or treated with $\mathrm{K}-350(5 \mu \mathrm{M})$ for the indicated times. H3: total histone H3 as a control; AcH3-K14: acetylated Lys 14 of histone $\mathrm{H} 3$.

A

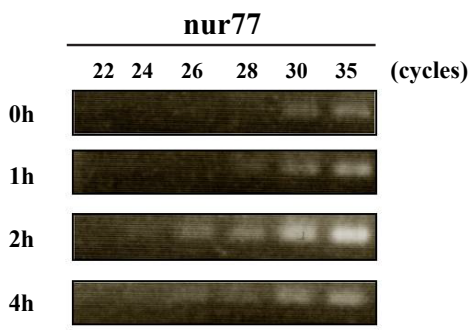

B

C
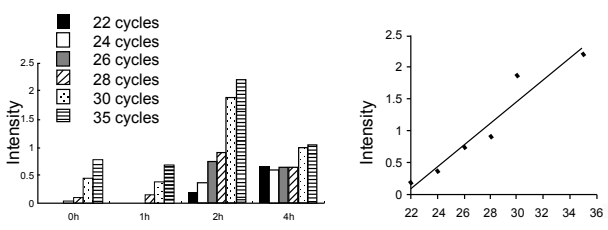

cycles

Figure 4: Treatment of PC12 cells with $\mathrm{K}-350$ induces nur77 gene expression. (A) PC12 cells were treated with $5 \mu \mathrm{M}$ K-350 for the indicated times in serumfree DMEM. nur77 and beta actin (control) mRNAs were detected by RT-PCR. (B) Semiquantitative analysis of nur77 gene expression. For nur77 cDNAs, the total area of white pixels and their intensity were determined by using CS Analyzer 1.0 software. PCR amplification was performed for 22 (black), 24 (white), 26 (gray), 28 (stripe), 30 (dot) and 35 (horizontal stripe) cycles.

(C) Semiquantitative analysis of nur77 gene expression after treatment with $\mathrm{K}-350$ for $2 \mathrm{~h}$. 
Citation: Maruoka H, Hosokawa R, Hirata Y, Kawa H, Okamoto K, et al. (2012) New Orally Bioavailable 2-aminobenzamide-type Histone Deacetyase Inhibitor Promotes Neurite Outgrowth via histone H3 Modification in PC12 cells: a Possible Therapeutic Candidate for Neuronal Diseases. J Bioengineer \& Biomedical Sci S5:001. doi:10.4172/2155-9538.S5-001

Page 4 of 5

We previously reported that PC12 cell treatment with either dbcAMP or NGF induces the expression of the immediate early gene (IEG) nur77 [20]. Since PC12 cells transition from an undifferentiated state to a differentiated state $0-4 \mathrm{~h}$ after treatment with $\mathrm{K}-350, \mathrm{PC} 12$ cells were treated with $5 \mu \mathrm{M}$ of $\mathrm{K}-350$ for $0,1,2,4 \mathrm{~h}$ and changes in nur77 gene expression were analyzed by RT-PCR. As shown in Figure 4-A and Figure 4-B, nur77 gene expression peaked $2 \mathrm{~h}$ after the treatment with $\mathrm{K}-350$, in a pattern that was similar to that observed for acetylation of Lys 14 of histone H3. This observation indicates that nur77 gene expression is upregulated during the early period of neuronal differentiation, 0-2 h after the treatment with K-350. A quantitative analysis of nur77 gene expression was performed based on an apparent linear increase in expression from 22 to 30 PCR cycles after $2 \mathrm{~h}$ of K-350 addition (Figure 4-C). These results suggested that the upregulation of nur77 gene expression by K-350 is essential for the neuronal differentiation induced by K-350.

\section{Discussion}

Here we report evidence in supporting the concept of HDAC inhibitors as possible novel therapeutics for the treatment of TBI. The present results demonstrated that the 2-aminobenzamide-type HDAC inhibitor K-350 promotes neurite outgrowth in PC12 cells. Although it is known that HDAC inhibitors induce neurite outgrowth [16,17], this report is the first to show that K-350 induces neurite outgrowth. This result suggests that K-350 may be a beneficial for the treament of TBI.

Kiyokawa et al. [18] reported that K-350 can be administered orally, and that mice dosed with K-350 do not show an action abnormality when compared with mice dosed with MS-275. The authors also reported that K-350 inhibited the growth of the cancer cells via a mechanism similar to that of MS-275 [18]. From these results, K-350 was anticipated as being both useful and less toxic than other drugs. Conversely, TSA is known to promote neurite outgrowth in a manner similar to that observed for K-350. However, because TSA shows strong cytotoxicity, it is less likely to be developed as a therapeutic drug for human use. Importantly, the current results indicated that K-350 is less toxic than MS-275 in PC12 cells, suggesting that K-350 may be a viable compound for development as a therapeutic drug in the human nervous system.

In this report we show that treatment with $\mathrm{K}-350$ at concentrations up to $15 \mu \mathrm{M}$ caused minimal PC12cell death after $24 \mathrm{~h}$ in culture (Figure 1). On the other hand, Kiyokawa et al. [18] reported that K-350 exhibited a cell growth IC50 of $0.7 \mu \mathrm{M}$ in HCT116 cells. There are at least two possible explanations for the reported differential sensitivity of PC12 cells and HCT 116 cells to K-350. One possibility is a difference in the effects of $\mathrm{K}-350$ on the organ from which each cell line was derived. Specifically, the PC12 cell line was derived from a pheochromocytoma of the rat adrenal medulla, while HCT116 is a human colon adenocarcinoma cell line. Both the species and the organ differences could potentially cause the observed differential sensitivity to K-350. However, it is important to note that the effects of K-350 on each derived organ are largely unknown. Another possible explanation for the differential sensitivity is the difference in doubling time between the two cell lines. HCT 116 cells have a doubling time of approximately 1 day, while the doubling time for PC12 cells is around 2 days. Further studies are required to fully elucidate the detailed mechanisms of action for K-350.

We also report for the first time that K-350 induces acetylation of Lys 14 on histone $\mathrm{H} 3$ at $4 \mathrm{~h}$ (Figure 3 ) and neurite outgrowth at 24 $\mathrm{h}$ in PC12 cells (Figure 2). These results suggest that K-350-induced acetylation of histone $\mathrm{H} 3$ may be an important regulator of neurite outgrowth in PC12 cells. Currently, the specific subtype of HDAC that is inhibited by $\mathrm{K}-350$ has not been established. Further investigations into the genes controlled by the HDAC subtype(s) that are inhibited by $\mathrm{K}-350$ will likely be critical in order to elucidate the mechanism(s) of K-350-induced neurite outgrowth.

Furthermore, we have demonstrated that nur77 gene expression participates in K-350-induced neurite outgrowth (Figure.4). This report is the first to show that nur77 gene expression contributes to K-350 promotion of neurite outgrowth. However, the detailed mechanism by which K-350 regulates nur77 gene expression is currently unclear and will require further investigation.

Nur77 is an orphan nuclear steroid receptor that belongs to the steroid/thyroid hormone receptor superfamily of transcription factors [24]. Furthermore, the Nur77 protein also has a function quite distinct from transcription regulation: it translocates from the nucleus to mitochondria in order to initiate apoptosis [25]. It was recently demonstrated that Nur77 interacts with Bcl-2, inducing a conformational change in Bcl-2 and converting its function from a cell protective role to a more lethal role [26]. We previously reported that treatment of PC12 cells with dbcAMP induced nur77 gene expression and was associated with acetylation of histone H3 Lys14 [20]. These data suggest that nur77 is localized to the cell nucleus and functions in that compartment to promote neurite outgrowth and in contrast translocates to mitochondria in order to induce apoptosis.

In summary, our report revealed that K-350 induces neurite outgrowth and cell death in higher does (Figure 1 and Figure 2). These data suggest that the Nur77 protein is translocated from the nucleus to mitochondria induced by K-350. However, the detailed mechanism is unknown. It is thought that the role of Nur77 in K-350 induced neurite outgrowth and cell death in higher doses correlates with the translocation of Nur77 from the nucleus to the mitochondria. Further study will be required to elucidate this regulatory function.

The results of this study suggest that the expression of nur77, an essential immediate early gene for cell differentiation, is up-regulated during K-350-induced neurite outgrowth in PC12 cells. Further studies are necessary to gain insight into the detailed mechanisms regulating epigenetic modifications and related changes in gene expression during K-350-induced neurite outgrowth.

\section{Acknowledgments}

This research was supported, in part, by grants-in-aid for scientific research from MEXT (Ministry of Education, Culture, Sports, Science and Technology of Japan), HAITEKU (2002-2006) and SENRYAKU (2008-2012) from MEXT, and the Kansai University Special Research Funds, 2007 and 2009. This work was also supported, in part, by the Cooperative Research Program of the Institute for Protein Research, Osaka University.

\section{References}

1. Hauschild A, Trefzer U, Garbe C, Kaehler KC, Ugurel S, et al. (2008) Multicenter phase II trial of the histone deacetylase inhibitor pyridylmethyl-N\{4-[(2-aminophenyl)-carbamoyl]-benzyl\}-carbamate in pretreated metastatic melanoma. Melanoma Res 18: 274-278.

2. Camelo S, Iglesias AH, Hwang D, Due B, Ryu H, et al. (2005) Transcriptional therapy with the histone deacetylase inhibitor trichostatin $A$ ameliorates experimental autoimmune encephalomyelitis. J Neuroimmunol 164: 10-21.

3. Gerstner T, Bell N, Konig S (2008) Oral valproic acid for epilepsy--long-term experience in therapy and side effects. Expert Opin Pharmacother 9: 285-292.

4. Faraco G, Pancani T, Formentini L, Mascagni P, Fossati G, et al. (2006) Pharmacological inhibition of histone deacetylases by suberoylanilide 
Citation: Maruoka H, Hosokawa R, Hirata Y, Kawa H, Okamoto K, et al. (2012) New Orally Bioavailable 2-aminobenzamide-type Histone Deacetyase Inhibitor Promotes Neurite Outgrowth via histone H3 Modification in PC12 cells: a Possible Therapeutic Candidate for Neuronal Diseases. J Bioengineer \& Biomedical Sci S5:001. doi:10.4172/2155-9538.S5-001

hydroxamic acid specifically alters gene expression and reduces ischemic injury in the mouse brain. Mol Pharmacol 70: 1876-1884.

5. Kim HJ, Rowe M, Ren M, Hong JS, Chen PS, et al. (2007) Histone deacetylase inhibitors exhibit anti-inflammatory and neuroprotective effects in a rat permanent ischemic model of stroke: multiple mechanisms of action. $J$ Pharmacol Exp Ther 321: 892-901.

6. Ren M, Leng $Y$, Jeong M, Leeds PR, Chuang DM (2004) Valproic acid reduces brain damage induced by transient focal cerebral ischemia in rats: potential roles of histone deacetylase inhibition and heat shock protein induction. $J$ Neurochem 89: 1358-1367.

7. Ferrante RJ, Kubilus JK, Lee J, Ryu H, Beesen A, et al. (2003) Histone deacetylase inhibition by sodium butyrate chemotherapy ameliorates the neurodegenerative phenotype in Huntington's disease mice. J Neurosci 23: 9418-9427.

8. Gardian G, Browne SE, Choi DK, Klivenyi P, Gregorio J, et al. (2005) Neuroprotective effects of phenylbutyrate in the $\mathrm{N} 171-82 \mathrm{Q}$ transgenic mouse model of Huntington's disease. J Biol Chem 280: 556-563.

9. Simonini MV, Camargo LM, Dong E, Maloku E, Veldic M, et al. (2006) The benzamide MS-275 is a potent, long-lasting brain region-selective inhibitor of histone deacetylases. Proc Natl Acad Sci U S A 103: 1587-1592.

10. Saha RN, Pahan K (2006) HATs and HDACs in neurodegeneration: a tale of disconcerted acetylation homeostasis. Cell Death Differ 13: 539-550.

11. Schwechter BR, Millet LE, Levin LA (2007) Histone deacetylase inhibitionmediated differentiation of RGC-5 cells and interaction with survival. Invest Ophthalmol Vis Sci 48: 2845-2857.

12. Siebzehnrubl FA, Buslei R, Eyupoglu IY, Seufert S, Hahnen E, et al. (2007) Histone deacetylase inhibitors increase neuronal differentiation in adult forebrain precursor cells. Exp Brain Res 176: 672-678.

13. Balasubramaniyan V, Boddeke E, Bakels R, Kust B, Kooistra S, et al. (2006) Effects of histone deacetylation inhibition on neuronal differentiation of embryonic mouse neural stem cells. Neuroscience 143: 939-951.

14. Kanai H, Sawa A, Chen RW, Leeds P, Chuang DM (2004) Valproic acid inhibits histone deacetylase activity and suppresses excitotoxicity-induced GAPDH nuclear accumulation and apoptotic death in neurons. Pharmacogenomics $\mathrm{J} 4$ 336-344.

15. Leng Y, Chuang DM (2006) Endogenous alpha-synuclein is induced by valproic acid through histone deacetylase inhibition and participates in neuroprotection against glutamate-induced excitotoxicity. J Neurosci 26: 7502-7512.

16. Inokoshi J, Katagiri M, Arima S, Tanaka H, Hayashi M, et al. (1999) Neuronal differentiation of neuro 2a cells by inhibitors of cell cycle progression, trichostatin A and butyrolactone I. Biochem Biophys Res Commun 256: 372-376.

17. Gaub P, Tedeschi A, Puttagunta R, Nguyen T, Schmandke A, et al. (2010) HDAC inhibition promotes neuronal outgrowth and counteracts growth cone collapse through CBP/p300 and P/CAF-dependent p53 acetylation. Cell Death Differ 17: 1392-1408.

18. Kiyokawa S, Hirata Y, Nagaoka Y, Shibano M, Taniguchi M, et al. (2010) New orally bioavailable 2-aminobenzamide-type histone deacetylase inhibitor possessing a (2-hydroxyethyl)(4-(thiophen-2-yl)benzyl)amino group. Bioorg Med Chem 18: 3925-3933.

19. Hao Y, Creson T, Zhang L, Li P, Du F, et al. (2004) Mood stabilizer valproate promotes ERK pathway-dependent cortical neuronal growth and neurogenesis. J Neurosci 24: 6590-6599.

20. Maruoka H, Sasaya H, Shimamura $Y$, Nakatani $Y$, Shimoke $K$, et al. (2010) Dibutyryl-cAMP up-regulates nur77 expression via histone modification during neurite outgrowth in PC12 cells. J Biochem 148: 93-101.

21. Shimoke K, Kishi S, Utsumi T, Shimamura Y, Sasaya H, et al. (2005) NGF induced phosphatidylinositol 3-kinase signaling pathway prevents thapsigargintriggered ER stress-mediated apoptosis in PC12 cells. Neurosci Lett 389: 124 128

22. Freeman WM, Brebner K, Lynch WJ, Patel KM, Robertson DJ, et al. (2002) Changes in rat frontal cortex gene expression following chronic cocaine. Brain Res Mol Brain Res 104: 11-20.

23. Chan SL, Fu W, Zhang P, Cheng A, Lee J, et al. (2004) Herp stabilizes neuronal $\mathrm{Ca}^{2+}$ homeostasis and mitochondrial function during endoplasmic reticulum stress. J Biol Chem 279: 28733-28743.

24. Hsu HC, Zhou T, Mountz JD (2004) Nur77 family of nuclear hormone receptors. Curr Drug Targets Inflamm Allergy 3: 413-423.

25. Jeong JH, Park JS, Moon B, Kim MC, Kim JK, et al. (2003) Orphan nuclea receptor Nur77 translocates to mitochondria in the early phase of apoptosis induced by synthetic chenodeoxycholic acid derivatives in human stomach cancer cell line SNU-1. Ann N Y Acad Sci 1010: 171-177.

26. Jacobs CM, Boldingh KA, Slagsvold HH, Thoresen GH, Paulsen RE (2004) ERK2 prohibits apoptosis-induced subcellular translocation of orphan nuclear receptor NGFI-B/TR3. J Biol Chem 279: 50097-50101.
This article was originally published in a special issue, Bioengineeering Techniques handled by Editor(s). Dr. Partha Roy, "University of Pittsburgh", USA. 